\title{
Genetic diversity of Cryptosporidium identified in clinical samples from cities in Brazil and Argentina
}

\author{
Regina Helena Saramago Peralta ${ }^{1 /+}$, Jorge Néstor Velásquez ${ }^{2}$, Flavia de Souza Cunha ${ }^{1}$, \\ María Laura Pantano ${ }^{3}$, Fernando Campos Sodré1, Sidnei da Silva ${ }^{4}$, \\ Osvaldo Germán Astudillo3 ${ }^{3}$, José Mauro Peralta ${ }^{5}$, Silvana Carnevale ${ }^{3,6}$
}

\author{
${ }^{1}$ Universidade Federal Fluminense, Faculdade de Medicina, Departamento de Patologia, Niterói, RJ, Brasil \\ ${ }^{2}$ Hospital Municipal de Infecciosas Dr Francisco Javier Muñiz, Buenos Aires, Argentina ${ }^{3}$ Instituto Nacional de Enfermedades Infecciosas, \\ Administración Nacional de Laboratorios e Institutos de Salud Dr Carlos G Malbrán, Buenos Aires, Argentina \\ ${ }^{4}$ Fundação Oswaldo Cruz, Instituto Nacional de Infectologia Evandro Chagas, Laboratório de Parasitologia, Rio de Janeiro, RJ, Brasil \\ ${ }^{5}$ Universidade Federal do Rio de Janeiro, Instituto de Microbiologia Prof Paulo de Góes, Rio de Janeiro, RJ, Brasil \\ ${ }^{6}$ Consejo Nacional de Investigaciones Científicas y Técnicas, Buenos Aires, Argentina
}

The identification and characterisation of Cryptosporidium genotypes and subtypes are fundamental to the study of cryptosporidiosis epidemiology, aiding in prevention and control strategies. The objective was to determine the genetic diversity of Cryptosporidium in samples obtained from hospitals of Rio de Janeiro, Brazil, and Buenos Aires, Argentina. Samples were analysed by microscopy and TaqMan polymerase chain reaction (PCR) assays for Cryptosporidium detection, genotyped by nested-PCR-restriction fragment length polymorphism (RFLP) analysis of the 18S rRNA gene and subtyped by DNA sequencing of the gp60 gene. Among the 89 samples from Rio de Janeiro, Cryptosporidium spp were detected in 26 by microscopy/TaqMan PCR. In samples from Buenos Aires, Cryptosporidium was diagnosed in 15 patients of the 132 studied. The TaqMan PCR and the nested-PCR-RFLP detected Cryptosporidium parvum, Cryptosporidium hominis, and co-infections of both species. In Brazilian samples, the subtypes IbA10G2 and IIcA5G3 were observed. The subtypes found in Argentinean samples were IbA10G2, IaA10G1R4, IaA11G1R4, and IeA11G3T3, and mixed subtypes of Ia and II families were detected in the co-infections. C. hominis was the species more frequently detected, and subtype family Ib was reported in both countries. Subtype diversity was higher in Buenos Aires than in Rio de Janeiro and two new subtypes were described for the first time.

Key words: Cryptosporidium - genotypes - gp60 subtypes - PCR

Cryptosporidium infections in immunocompromised individuals can be asymptomatic or can have severe clinical symptoms, such as profuse diarrhoea, which is usually accompanied by weight loss, malabsorption syndrome, and cholangitis (Putignani \& Menichella 2010). In the last two decades, some studies have described a significant risk factor for acquiring intestinal parasitic infections among human immunodeficiency virus (HIV)-infected patients compared with non-HIV controls. Cryptosporidiosis has a high prevalence among intestinal protozoa (Moura et al. 1989, Mohammad et al. 2004, Akinbo et al. 2010).

Laboratory diagnosis of cryptosporidiosis is usually performed by identification of oocysts in stools using an acid-fast stain, but this does not allow species identification, as they are morphologically indistinguishable (Chalmers \& Katzer 2013). The identification and characterisation of Cryptosporidium species and population

doi: 10.1590/0074-02760150303

Financial support: CNPq, FAPERJ, FOCANLIS (2011)

+ Corresponding author: peralta@micro.ufrj.br

Received 11 August 2015

Accepted 8 December 2015 variants (genotypes and subtypes) are fundamental to the study of cryptosporidiosis epidemiology and are useful in prevention and control strategies (Putignani $\&$ Menichella 2010). Because the oocysts of many species are indistinguishable from one another, molecular methods are essential for identification of the species, genotype, and subtype of Cryptosporidium in order to specifically identify the organism responsible for the infection and the source and routes of transmission. The taxonomy of Cryptosporidium has been standardised with a guideline that includes morphometric data on oocysts, genetic characterisation, natural, and when feasible, experimental host specificity, and compliance with International Commission on Zoological Nomenclature rules (Ryan et al. 2014).

The methods currently used for detection and species characterisation of Cryptosporidium are based on nested-polymerase chain reaction (PCR), PCR-restriction fragment length polymorphism (RFLP), and real-time PCR (Xiao 2010). The genetic markers used are the gene encoding for $18 \mathrm{~S}$ ribosomal subunit, the COWP gene, encoding a protein of the oocyst wall, the $h s p 70$ gene, which encodes a heat shock protein, internal transcribed spacer (ITS)-1 and ITS-2, the TRAP gene, and the gene encoding the glycoproteins GP60 and GP40 (Xiao 2010, Navarro-i-Martinez et al. 2011, Galván et al. 2014). There is no standard genetic locus recommended for species identity, but RFLP or sequencing of the 18S rRNA gene 
provides information about more species than the COWP gene locus (Muthusamy et al. 2006). Recent work has confirmed the utility of $g p 60$ sequencing and mini and microsatellite markers in the study of the population structure of Cryptosporidium and in understanding the transmission dynamics of infection (Feng et al. 2014). Currently, nearly 20 Cryptosporidium species and genotypes have been reported in humans, including Cryptosporidium hominis, Cryptosporidium parvum, Cryptosporidium meleagridis, Cryptosporidium felis, Cryptosporidium canis, Cryptosporidium cuniculus, Cryptosporidium ubiquitum, Cryptosporidium viatorum, Cryptosporidium muris, Cryptosporidium suis, Cryptosporidium fayeri, Cryptosporidium andersoni, Cryptosporidium bovis, Cryptosporidium scrofarum, Cryptosporidium tyzzeri, Cryptosporidium erinacei and Cryptosporidium I genotypes from horse, skunk, and chipmunk (Xiao 2010, Liu et al. 2014, Ryan \& Hijjawi 2015).

In Brazil and Argentina, the prevalence of human cryptosporidiosis varies widely range between $7-24 \%$ (Velásquez et al. 1997, Bachur et al. 2008, Barboni et al. 2008, Cardoso et al. 2011, Assis et al. 2013). However, there are few molecular studies of Cryptosporidium species and genotypes in humans in these countries where reports about subtypes of Cryptosporidium are not described (Carnevale et al. 2010, Meireles 2010, Velásquez et al. 2010, Rolando et al. 2012). Therefore, in the present study, we detected and characterised Cryptosporidium species and identified subtypes for the first time in faecal and/or biopsy samples from HIV+ individuals (adults and children) seeking medical attention in public hospitals in the cities of Rio de Janeiro, Brazil, and Buenos Aires, Argentina.

\section{SUBJECTS, MATERIALS AND METHODS}

Sample collection and DNA extraction in Rio de Janeiro - Ages of the included patients ranged from 20 75 years for adults and one-16 years for children, with a male/female ratio of 59/30. Eighty-nine faecal samples from HIV-infected patients with diarrhoea (82 adults and 7 children of both sexes) were screened for intestinal parasite infections, with special attention to Cryptosporidium spp, using a centrifuge-sedimentation technique, with two centrifugations of 2,000 $\mathrm{g} / 2$ min (Ritchie 1948). The 89 stool samples were collected from 2009-2013 in three different public hospitals in Rio de Janeiro, originating from Antônio Pedro University Hospital [Faculty of Medicine, Fluminense Federal University (UFF)] $(\mathrm{n}=77)$, Jesus Municipal Hospital $(\mathrm{n}=$ 7), and Evandro Chagas Institute of Clinical Research (Oswaldo Cruz Foundation) $(\mathrm{n}=5)$. Only samples with a medical request for the detection of Cryptosporidium spp were included in this study.

For Cryptosporidium oocyst identification, stool samples were subjected to a modified acid-fast staining technique (Ma \& Soave 1983). After parasitological exams, all samples were stored at $-20^{\circ} \mathrm{C}$ until molecular characterisation. This study was conducted with the approval of the Ethical Review Committee for Research of the Faculty of Medicine, UFF, protocol 020/07.

Total genomic DNA was extracted from $300 \mathrm{~mL}$ of stool samples using FastDNA ${ }^{\circledR}$ kit (MP Biomedicals) according to the manufacturer's protocol. Samples were disrupted in an FP120 cell disruptor (MP Biomedicals) at a speed of $5.5 \mathrm{~m} / \mathrm{s}$ for $10 \mathrm{~s}$. Potential inhibitors were removed by further purification using QIAquick PCR purification kit (Qiagen), following the manufacturer's instructions, and DNA extracts were stored at $-20^{\circ} \mathrm{C}$ until further processing.

Sample collection and DNA extraction in Buenos Aires - The study population consisted of 132 adult patients of both sexes (113 male/19 female) diagnosed with HIV who attended the Francisco J Muñiz and José María Penna Hospitals complaining of diarrhoea. Serial stool samples from each patient were processed for parasite analysis by examination of wet mount and slides stained using the modified Ziehl-Neelsen technique for cryptosporidia (Henriksen \& Pohlenz 1981), and Weber trichrome (Weber et al. 1992) and Gram-chromothrope (Moura et al. 1996), for microsporidia spores. DNA purification from stool samples was carried out by phenol-chloroform extraction according to Velásquez et al. (2010). Briefly, $1 \mathrm{~mL}$ of each sample was treated with $200 \mathrm{~mL}$ of ether and centrifuged for $5 \mathrm{~min}$ at $15,000 \mathrm{~g}$. The pellet was re-suspended in $1 \mathrm{~mL}$ of $70 \%$ ethanol and stored overnight at $-20^{\circ} \mathrm{C}$. Then, the sample was centrifuged for $3 \mathrm{~min}$ at $15,000 \mathrm{~g}$, washed twice with $1 \mathrm{~mL}$ of bidistilled water by centrifugation for $3 \mathrm{~min}$ at $15,000 \mathrm{~g}$, and dried at $37^{\circ} \mathrm{C}$. The pellet was incubated for $45 \mathrm{~min}$ at $4^{\circ} \mathrm{C}$ in $1 \mathrm{~mL}$ of sodium hypochlorite $(33 \mathrm{~g} / \mathrm{L})$. After this step, the material was centrifuged and washed as previously described. The pellet was then re-suspended in $200 \mu \mathrm{L}$ of phosphate-buffered saline pH 8 with $20 \mu \mathrm{L}$ of $5 \%$ trypsin and incubated overnight at $37^{\circ} \mathrm{C}$ at $200 \mathrm{rpm}$. All of the following steps were performed as previously described (Velásquez et al. 2010). Biopsy samples from the duodenum were collected by upper gastrointestinal endoscopy, fixed in formaldehyde, paraffin-embedded and stained with Giemsa and haematoxylin-eosin (Carey et al. 2004). Another biopsy specimen was collected in saline solution, stored at $-20^{\circ} \mathrm{C}$ and employed for DNA extraction and purification according to Velásquez et al. (2010). The research protocol was approved by the Ethical Committee for Research, Hospital Francisco J Muñiz, protocol 274.

The DNA recovery was above $10 \mathrm{pg}$, measured in a Qubit $^{\circledR}$ fluorometer (Invitrogen, USA).

TaqMan PCR assays - The TaqMan PCR procedure combined a duplex reaction for the detection of Cryptosporidium species and C. parvum and a simple reaction for the detection of $C$. hominis, as described previously (Jothikumar et al. 2008). The assays were performed with a 7500 Real-Time PCR System (Life Technologies, USA). Each $20-\mu \mathrm{L}$ duplex reaction (to identify Cryptosporidium species and C. parvum) contained $10 \mu \mathrm{L}$ of 2X Platinum Quantitative PCR SuperMix-UDG (Invitrogen), $100 \mathrm{nM}$ of each probe (JVAP 18S and JVAGP2), $250 \mathrm{nM}$ of each primer (JVAF, JVAR, JVAGF, and JVA$\mathrm{GR}$ ), and $5 \mu \mathrm{L}$ of DNA. For the $C$. hominis assay, each $20-\mu \mathrm{L}$ reaction contained $10 \mu \mathrm{L}$ of $2 \mathrm{X}$ Platinum Quantitative PCR SuperMix-UDG (Invitrogen), $250 \mathrm{nM}$ of each primer (JVAF, JVAR, JVAGF, and JVAGR), $5 \mathrm{mM}$ 
$\mathrm{MgCl}$, twice the probe concentration used for the duplex assay (200 nM), and $5 \mu \mathrm{L}$ of DNA. The Cryptosporidium PCR cycling conditions consisted of denaturation at $95^{\circ} \mathrm{C}$ for $2 \mathrm{~min}$ followed by 45 cycles of denaturation at $94^{\circ} \mathrm{C}$ for $10 \mathrm{~s}$, annealing at $55^{\circ} \mathrm{C}$ for $30 \mathrm{~s}$, and extension at $72^{\circ} \mathrm{C}$ for $20 \mathrm{~s}$. All assays included positive controls (C. hominis and C. parvum) and negative controls (DNA extracted from faecal samples negative for any parasites). To investigate the presence of inhibitory substances, negative samples were contaminated with around $10 \mathrm{fg}$ of Cryptosporidium DNA and subsequently submitted to TaqMan PCR.

Genotyping analysis - Cryptosporidium species were determined by nested PCR amplifying a 214 bp fragment of the small-subunit 18S rRNA gene and RFLP analysis of the secondary PCR products, using the restriction enzymes TaqI (Fermentas, Lifescience) and $V_{s p} \mathrm{I}$ (Fermentas, Life Science). Primers and amplification conditions used for PCR-RFLP were adopted from previous publications (Coupe et al. 2005). Reaction mixtures were prepared according to Velásquez et al. (2010), employing $10 \mathrm{X}$ buffer containing (NH4) ${ }_{2} \mathrm{SO}_{4}$, and adding $400 \mathrm{ng} /$ $\mu \mathrm{L}$ of bovine serum albumin. Cycle conditions were as follows: one cycle of $94^{\circ} \mathrm{C}$ for $5 \mathrm{~min}, 39$ cycles of a denaturation step at $94^{\circ} \mathrm{C}$ for $30 \mathrm{~s}$, an annealing step at $60^{\circ} \mathrm{C}$ $\left(58^{\circ} \mathrm{C}\right.$ for the $2 \mathrm{nd}$ round) for $45 \mathrm{~s}$, and an extension step at $72^{\circ} \mathrm{C}$ for $90 \mathrm{~s}$, with a final extension for $10 \mathrm{~min}$ at $72^{\circ} \mathrm{C}$.

Enzymatic-digested products were fractionated on a $2 \%$ agarose gel and visualised by ethidium bromide staining. All diagnoses were confirmed by RFLP analysis of additional, independent PCR products from the same sample.

Samples that contained C. parvum and C. hominis were further subtyped by DNA sequencing of the gp60 gene amplified by a nested PCR following the protocol described by Glaberman et al. (2002). Each sample was amplified at least three times by PCR. Primers AL3531 and AL3533 were used in the primary PCR and primers AL3532 and LX0029 in the secondary PCR. gp60 products were purified according to the manufacturer's instructions using a NucleoSpin ${ }^{\circledR}$ Extract II kit (MACHEREY-NAGEL GmbH and Co KG, Germany). Sequencing was carried out in both directions by the sequencing services of Institute of Biophysics Carlos Chagas, Macromolecular Metabolism Laboratory, Federal University of Rio de Janeiro (UFRJ), and Medical Biochemistry Institute, SONDA Laboratory, UFRJ, for the Brazilian samples, and by Macrogen Service, for the Argentinean ones. The nucleotide sequences obtained in this study were aligned with reference sequences retrieved from GenBank and gp60 sequences. The resulting sequences were edited and aligned with the Bioedit Sequence Alignment Editor 7.0.5.3.

\section{RESULTS}

Samples from Brazil - Thirty faecal samples were parasitologically positive in the exams and Cryptosporidium sp. oocysts were observed in 17 (19.1\%) samples. The other parasites detected in these samples were Strongyloides stercoralis (2.2\%), Giardia duodenalis (2.2\%), Entamoeba coli (1.1\%), Entamoeba histolytica/Entamoeba dispar complex (2.2\%), Blastocystis sp. (5.6\%), and Cystoisos- pora belli $(1.1 \%)$. The results of the dual TaqMan PCR procedure for the stool samples from patients are shown in Table I. The 18S rRNA TaqMan assay detected Cryptosporidium in $23(25.8 \%)$ samples. Four samples were amplified in the C. parvum assay, 14 samples were amplified as $C$. hominis, one sample was a mixed infection with $C$. hominis and C. parvum, and four samples were amplified only as a Cryptosporidium spp. Three samples previously determined to be Cryptosporidium-positive for microscopy were Cryptosporidium-negative using the TaqMan assay. For PCR inhibition control, these samples were contaminated with a known concentration of Cryptosporidium DNA and retested by PCR. All spiking samples tested had DNA amplification showing no inhibition effect. Species and subtypes of all 26 samples considered Cryptosporidium-positive by TaqMan assay and/or microscopy were analysed by nested-PCR-RFLP for 18S rRNA and nested-PCR for gp60 (Table I). Eight of these samples did not amplify during $18 \mathrm{~S}$ rRNA PCR or $g p 60$ PCR and two amplified only in gp60 PCR. Nine samples were negative in microscopy and four amplified only for Cryptosporidium spp in the TaqMan PCR assay. The RFLP confirmed the result from the TaqMan assay, including the result with a mixed infection. The subtype IbA10G2 was observed in 13 C. hominis samples and was the only subtype found in this studied population. In four samples presented C. parvum, one was sequenced in which the subtype IIcA5G3 was found. The presence of C. parvum was observed only in the children group (4/7).

Samples from Argentina - The ages of 132 patients who participated in this study ranged from 20-50 years. Faecal and/or biopsy samples from 31 patients were positive for parasites and 14 for microsporidia. Cryptosporidium was diagnosed in 15 patients (11.4\%). The other parasites detected were C. belli $(9.8 \%), G$. duodenalis (5.3\%), S. stercoralis (3\%), and Cyclospora cayetanensis $(0.8 \%)$. Those 15 Cryptosporidium-positive samples were analysed by real-time PCR assays and the results are shown in Table II. Five samples were amplified in the $C$. parvum assay, nine samples were amplified for $C$. hominis, and one sample was co-infected with C. hominis and C. parvum. Species of the 15 Cryptosporidiumpositive samples were analysed by nested-PCR-RFLP for 18S rRNA, revealing nine $C$. hominis infections, four C. parvum infections, and two co-infections of both species. The results confirmed the species determined by real-time PCR, with the exception of another case of coinfection that had been detected as C. parvum only. The subtypes were analysed by amplification and sequencing of the gp60 gene in eight samples obtained from biopsy. The subtypes for $C$. hominis were IbA10G2 in three samples and IaA10G1R4, IaA11G1R4, and IeA11G3T3 in one sample each. In two samples with co-infections, mixed subtypes of Ia and IIa were detected.

\section{DISCUSSION}

In the present study, the diversity of Cryptosporidium parasites from patients living in the cities of Buenos Aires and Rio de Janeiro was examined. Data on specific genotypes involved in human infections in the region are 


\section{TABLE I}

Results of microscopic examination, TaqMan polymerase chain reaction (PCR), PCR-restriction fragment length polymorphism (RFLP) $18 \mathrm{~S}$ rDNA, and by gp60 PCR/sequencing of faecal samples from hospitals of the city of Rio de Janeiro, Brazil, for Cryptosporidium characterisation

\begin{tabular}{|c|c|c|c|c|}
\hline Sample & Microscopy & Real-time PCR & 18S rDNA/RFLP & gp60/sequencing \\
\hline Pc1 & Cryptosporidium spp & C. hominis & C. hominis & IbA10G2 \\
\hline Pc2 & Cryptosporidium spp & C. hominis & C. hominis & IbA10G2 \\
\hline Pc6 & Negative & C. hominis & No amplification & No amplification \\
\hline Pc7 & Negative & C. hominis & C. hominis & IbA10G2 \\
\hline Pc8 & Negative & Cryptosporidium spp & No amplification & No amplification \\
\hline Pc13 & Negative & Cryptosporidium spp & No amplification & No amplification \\
\hline Pc16 & Cryptosporidium spp & Cryptosporidium spp & No amplification & No amplification \\
\hline Pc17 & Cryptosporidium spp & No amplification & No amplification & No amplification \\
\hline Pc20 & Cryptosporidium spp & Cryptosporidium spp & C. hominis & IbA10G2 \\
\hline Pc27 & Negative & C. hominis & C. hominis & IbA10G2 \\
\hline Pc28 & Negative & C. parvum & No amplification & No amplification \\
\hline $\operatorname{Pc} 44$ & Cryptosporidium spp & No amplification & No amplification & No amplification \\
\hline Pc46 & Cryptosporidium spp & No amplification & No amplification & No amplification \\
\hline Pc52 & Negative & C. hominis & C. hominis & IbA10G2 \\
\hline Pc78 & Cryptosporidium spp & C. hominis & C. hominis & IbA10G2 \\
\hline Pc79 & Cryptosporidium spp & C. hominis & C. hominis & IbA10G2 \\
\hline Pc80 & Cryptosporidium spp & C. hominis & C. hominis & Cryptosporidium \\
\hline Pc81 & Cryptosporidium spp & C. hominis & C. hominis & IbA10G2 \\
\hline Pc82 & Cryptosporidium spp & C. hominis & C. hominis & IbA10G2 \\
\hline Pc83 & Cryptosporidium spp & C. hominis & C. hominis & IbA10G2 \\
\hline Pc84 & Cryptosporidium spp & C. hominis & C. hominis & IbA10G2 \\
\hline Pc85 & Cryptosporidium spp & C. hominis & C. hominis & Cryptosporidium \\
\hline Pc86 & Negative & C. parvum & No amplification & IbA10G2 \\
\hline Pc87 & Negative & C. parvum & No amplification & Cryptosporidium \\
\hline Pc88 & Cryptosporidium spp & C. parvum/C. hominis & C. parvum/C. hominis & Cryptosporidium \\
\hline Pc89 & Cryptosporidium spp & C. parvum & C.parvum & IIcA5G3 \\
\hline
\end{tabular}

still limited, and to the best of our knowledge, this is the first report on the study of the Cryptosporidium subtypes infecting humans performed in Argentina and Brazil.

The results showed that $C$. hominis was detected more frequently than C. parvum in HIV-infected patients from both areas, but co-infections of both species were also present. C. parvum and C. hominis have been reported as the most common species causing infections in HIV-infected people in developed countries [France, Switzerland, Spain, and the United States of America (USA)] and developing countries (Jamaica, Kenya, Malaysia, Peru, Portugal, South Africa, South India, Thailand, and Vietnam) (Gatei et al. 2003, Zaidah et al. 2008). The results of the present work appear to be consistent with those in Peru, Australia, Kenya, South Africa, Thailand, the USA, and Vietnam, where $C$. hominis is reported to be the more common causative agent of cryptosporidiosis in immunocompromised people (Cama et al. 2007, Widmer \& Sullivan 2012). Cryptosporidium subtyping at the gp60 level in HIV infected patients has been carried out in a few countries (Xiao \& Feng 2008).

Different types of molecular techniques have been used in the differentiation of Cryptosporidium species/ genotypes, with the $S S U$ rRNA-based tools being the most employed, especially PCR-RFLP (Xiao 2010). The use of this gene is mainly due to the presence of semi-conserved and hyper-variable regions in a multi-copy. In our study, we applied this technique for species identification, and it was also useful for co-infection detection. All positive samples by microscopy were analysed by a previously described TaqMan PCR assay that allowed differentiation of $C$. hominis and C. parvum at the species level. According to our results, both methods could be employed simultaneously to improve and confirm the results. Molecular methods for detecting Cryptosporidium in clinical specimens have been shown to be more sensitive than conventional microscopy (Chalmers \& Katzer 2013, Yang et al. 2013). The TaqMan procedure had a specificity of $94 \%$ for detecting Cryptosporidium in clinical specimens and a sensitivity that is better than conventional microscopy and comparable to other molecular methods used for confirmatory identification of Cryptosporidium species (Jothikumar et al. 2008).

Of four samples that amplified only as a Cryptosporidium spp in TaqMan PCR, one also amplified in nested-PCR gp60 and the subtype was defined as 


\section{TABLE II}

Molecular analyses of Cryptosporidium spp positive faecal and biopsy samples from the city of Buenos Aires, Argentina

\begin{tabular}{|c|c|c|c|}
\hline Samples & $\begin{array}{l}\text { Real-time } \\
\text { PCR }\end{array}$ & $\begin{array}{l}\text { 18S rDNA/ } \\
\text { RFLP }\end{array}$ & $\begin{array}{c}g p 60 / \\
\text { sequencing }\end{array}$ \\
\hline 1 & C. parvum & C. parvum & NP \\
\hline 2 & C. parvum & C. parvum & NP \\
\hline 4 & C. hominis & C. hominis & NP \\
\hline 6 & C. hominis & C. hominis & IbA10G2 \\
\hline 21 & C. hominis & C. hominis & IbA10G2 \\
\hline 22 & C. hominis & C. hominis & IbA10G2 \\
\hline 23 & C. hominis & C. hominis & IaA10G1R4 \\
\hline 24 & C. hominis & C. hominis & IeA11G3T3 \\
\hline 25 & C. hominis & C. hominis & IaA11G1R4 \\
\hline 27 & $\begin{array}{l}\text { C. hominis/ } \\
\text { C. parvum }\end{array}$ & $\begin{array}{l}\text { C. parvum/ } \\
\text { C. hominis }\end{array}$ & Ia, IIa \\
\hline 28 & C. parvum & $\begin{array}{l}\text { C. hominis/ } \\
\text { C. parvum }\end{array}$ & Ia, IIa \\
\hline 39 & C. parvum & C. parvum & NP \\
\hline 46 & C. parvum & C. parvum & NP \\
\hline 47 & C. hominis & C. hominis & NP \\
\hline 50 & C. hominis & C. hominis & NP \\
\hline
\end{tabular}

NP: not performed; PCR: polymerase chain reaction; RFLP: restriction fragment length polymorphism.

IbA10G2. The others three samples were probably different species of Cryptosporidium and additional analysis is necessary for confirmation. We did not have success in amplifying the $18 \mathrm{~S}$ and gp60 gene in these samples.

Given that $C$. hominis and $C$. parvum were responsible for the Cryptosporidium infections in the HIV-infected patients studied here, a further evaluation of the genetic variation within these two species was carried out by DNA sequence analysis of the $60-\mathrm{kDa}$ glycoprotein gene. This single-copy gene has tandem repeats of the serine-coding trinucleotide TCA, TCG, or TCT at the 5 ' end of the gene, which vary in number, and there are extensive sequence differences in the nonrepeat regions, which categorise each species to several subtype families (Strong et al. 2000, Xiao 2010).

Until now, $C$. hominis had nine gp60 subtype families, Ia-Ij (Nichols et al. 2014). The subtype family Ib is dominant and broadly distributed, with more than $70 \%$ of records referencing this subtype (Jex \& Gasser 2010), followed by Ia, Id, and Ie, with less than $10 \%$ each. Additionally, there is variation in subtype richness, diversity, and distribution within each of the gp60 subtype families of $C$. hominis. The subtype family $\mathrm{Ib}$ is represented by nine subtypes, dominated by IbA10G2, which is distributed on all inhabited continents. This subtype has been implicated in some waterborne and foodborne outbreaks of human cryptosporidiosis (Sulaiman et al. 2001, Glaberman et al. 2002, Cohen et al. 2006, Widerström et al. 2014). Subtype IbA10G2 has been found in approximately $50 \%$ of $C$. hominis-associated outbreaks in the USA (Xiao \& Ryan 2004). In a longitudinal study in Lima, Peru, IbA10G2 was more virulent than other $C$. hominis subtypes (Cama et al. 2008).

In samples from Rio de Janeiro, only one subtype of C. hominis was identified, corresponding to IbA10G2. Instead, in samples from Buenos Aires, three C. hominis subtype families were detected (Ib, Ia, and Ie). The IbA10G2 was the predominant subtype, but three others were identified, namely IaA10G1R4, IaA11G1, and IeA11G3T3. Although the sample size is small, homogeneity within the $C$. hominis at the gp60 locus is unusual, as subtype families such as Ia and Id are usually equally abundant in most developing countries (Leav et al. 2002, Peng et al. 2003, Cama et al. 2008, Valenzuela et al. 2014). It has been shown that there are differences in the molecular epidemiology of $C$. hominis between developing and developed countries. In nonindustrialised countries, a greater variety of $C$. hominis subtypes have been reported with multiple subtype families and multiple subtypes within families Ia and Id. Much less genetic diversity is observed in $C$. hominis in some industrialised countries, where most $C$. hominis infections are caused by the Ib subtype family, with the majority of these cases having the IbA10G2 subtype (Xiao 2010). Recently, the subtype IbA10G2 was also prevalent in 161 cryptosporidiosis cases in two hospitals in Barcelona, Spain, corresponding to $90 \%$ of all C. hominis isolates (Segura et al. 2015). The presence of Ib and IIc subtype families in Brazil may suggest anthroponotic transmission, while the findings from Argentina suggest that zoonotic transmission may play a role.

Among the Ia subfamily, IaA12G1R1 is the most common subtype and has been reported in humans from Japan, Nepal, Pakistan, and Peru (Wu et al. 2003, Cama et al. 2008, Chalmers 2008). Among this subfamily, two subtypes were identified in the Argentinean samples, belonging to IaA10G1R4 (GenBank KT381976) and IaA11G1R4 (GenBank KT381977), and both have not been previously described.

Subtype family Ie has low subtype richness and diversity, as most Ie human infections in developing countries are caused by IeA11G3T3 (Sharma et al. 2013, Adamu et al. 2014, Valenzuela et al. 2014). Our study in Buenos Aires identified this subtype in one sample.

Regarding C. parvum, gp60 subgenotyping for Brazilian patients identified only the IIcA5G3 lineage in one sample. The subtype family IIa is globally distributed and the most common C. parvum infection in humans (Xiao $\&$ Feng 2008). The subtype families IIc and IId are also common and broadly distributed. The other known families, IIb and IIe-k, are rare (Jex \& Gasser 2010). Eibach et al. (2015) described that all identified $C$. parvum and $C$. hominis subtypes found in Brazilian human samples have not yet been identified in any animal samples, suggesting a dominant anthroponotic transmission in the region of Rio de Janeiro. These authors also found similar results in a rural region of Ghana. This type of transmission is expected in the urban region of Rio de Janeiro.

Genotyping by TaqMan PCR and 18S PCR-RFLP, and subtyping by gp60-based sequencing revealed mixed infections of Cryptosporidium species/subtypes in three samples. In one brazilian sample, $C$. hominis and C. par- 
vum were detected for both genotyping assays, but the gp60 subtyping was not possible to analyse. For the Argentinean samples, co-infection of C. hominis and C. parvum were detected in two cases, with the subtype families Ia and IIa. There are few records showing mixed infections at the subtyping level (Ajjampur et al. 2010, Sharma et al. 2013), and this is the first report for human samples from Argentina. The interference of the subtypes in the severity of the infection also beginning to be studied and the IbA10G2 subtype has been described as one of the most virulent among the subtypes of $C$. hominis ( $\mathrm{Li}$ et al. 2013). Subtype family IbA10G2 was reported in both countries, but great subtype diversity was detected in Argentina. So far, only two studies have reported subtyping of Cryptosporidium in South America, involving Peruvian children (Cama et al. 2008) and Peruvian HIV-infected persons (Cama et al. 2007). Differentiating the species and subtypes requires the use of high resolution molecular tools, and subtyping is important for understanding population structure. Further information from larger samples is necessary to provide insights on subtype lineages to elucidate the value of geographical/pathogenic variation in Cryptosporidium species/genotypes.

\section{REFERENCES}

Adamu H, Petros B, Zhang G, Kassa H, Amer S, Ye J, Feng Y, Xiao L 2014. Distribution and clinical manifestations of Cryptosporidium species and subtypes in HIV/AIDS patients in Ethiopia. PLoS Negl Trop Dis 8: e2831.

Ajjampur SS, Liakath FB, Kannan A, Rajendran P, Sarkar R, Moses PD, Simon A, Agarwal I, Mathew A, O'Connor R, Ward H, Kang G 2010. Multisite study of cryptosporidiosis in children with diarrhea in India. J Clin Microbiol 48: 2075-2081.

Akinbo FO, Okaka CE, Omorgie R 2010. Prevalence of intestinal parasitic infections among HIV patients in Benin city, Nigeria. Libyan J Med 5: 5506.

Assis DC, Resende DV, Cabrine-Santos M, Correia D, Oliveira-Silva MB 2013. Prevalence and genetic characterization of Cryptosporidium spp and Cystoisospora belli in HIV-infected patients. Rev Inst Med Trop Sao Paulo 55: 149-154.

Bachur TP, Vale JM, Coêlho IC, Queiroz TR, Chaves CS 2008. Enteric parasitic infections in HIV/AIDS patients before and after the highly active antiretroviral therapy. Braz J Infect Dis 12: 115-122.

Barboni G, Candi M, Villacé MI, Leonardelli A, Balbaryski J, Gaddi E 2008. Intestinal cryptosporidiosis in HIV infected children. Medicina (B Aires) 68: 213-218.

Cama VA, Bern C, Roberts J, Cabrera L, Sterling CR, Ortega Y, Gilman RH, Xiao L 2008. Cryptosporidium species and subtypes and clinical manifestations in children, Peru. Emerg Infect Dis 14: $1567-1574$.

Cama VA, Ross JM, Crawford S, Kawai V, Chavez-Valdez R, Vargas D, Vivar A, Ticona E, Navincopa M, Williamson J, Ortega Y, Gilman RH, Bern C, Xiao L 2007. Differences in clinical manifestations among Cryptosporidium species and subtypes in HIVinfected persons. J Infect Dis 196: 684-691.

Cardoso LV, Galisteu KJ, Schiesari Jr A, Chahla LA, Canille RM, Belloto MV, Franco C, Maia IL, Rossit AR, Machado RL 2011. Enteric parasites in HIV-1/AIDS-infected patients from a northwestern São Paulo reference unit in the highly active antiretroviral therapy era. Rev Soc Bras Med Trop 44: 665-669.

Carey CM, Lee H, Trevors JT 2004. Biology, persistence, and detection of Cryptosporidium parvum and Cryptosporidium hominis oocyst. Water Res 38: 818-862.
Carnevale S, Velásquez JN, Marta E, Astudillo OG, Etchart C, Chertcoff AV, Di Risio C 2010. Identification of Cryptosporidium hominis in a patient with sclerosing cholangitis and AIDS. Acta Gastroenterol Latinoam 40: 271-275.

Chalmers RM 2008. Cryptosporidium: from laboratory diagnosis to surveillance and outbreaks. Parasite 15: 372-378.

Chalmers RM, Katzer F 2013. Looking for Cryptosporidium: the application of advances in detection and diagnosis. Trends Parasitol 29: 237-251.

Cohen S, Dalle F, Gallay A, Di Palma M, Bonnin A, Ward HD 2006. Identification of Cpgp40/15 type Ib as the predominant allele in isolates of Cryptosporidium spp from a waterborne outbreak of gastroenteritis in south Burgundy, France. J Clin Microbiol 44: 589-591.

Coupe S, Sarfati C, Hamane S, Derouin F 2005. Detection of Cryptosporidium and identification to the species level by nested PCR and restriction fragment length polymorphism. J Clin Microbiol 43: 1017-1023.

Eibach D, Krumkamp R, Al-Emran HM, Sarpong N, Hagen RM, Adu-Sarkodie Y, Tannich E, May J 2015. Molecular characterization of Cryptosporidium spp among children in rural Ghana. PLoS Negl Trop Dis 6: 1-12.

Feng Y, Tiao N, Li N, Hlavsa M, Xiao L 2014. Multilocus sequence typing of an emerging Cryptosporidium hominis subtype in the United States. J Clin Microbiol 52: 524-530.

Galván AL, Magnet A, Izquierdo F, Vadillo CF, Peralta RH, Angulo S, Fenoy S, del Aguila C 2014. A year-long study of Cryptosporidium species and subtypes in recreational, drinking, and wastewater from the central area of Spain. Sci Total Environ 468-469: 368-375.

Gatei W, Greensill J, Ashford RW, Cuevas LE, Parry CM, Cunliffe NA, Beeching NJ, Hart CA 2003. Molecular analysis of the $18 \mathrm{~S}$ rRNA gene of Cryptosporidium parasites from patients with or without human immunodeficiency virus infections living in Kenya, Malawi, Brazil, the United Kingdom, and Vietnam. J Clin Microbiol 41: 1458-1462.

Glaberman S, Moore JE, Lowery CJ, Chalmers RM, Sulaiman I, Elwin K, Rooney PJ, Millar BC, Dooley JS, Lal AA, Xiao L 2002. Three drinking-water-associated cryptosporidiosis outbreaks, northern Ireland. Emerg Infect Dis 8: 631-633.

Henriksen SA, Pohlenz JF 1981. Staining of cryptosporidia by a modified Ziehl-Neelsen technique. Acta Vet Scand 22: 594-596.

Jex AR, Gasser RB 2010. Genetic richness and diversity in Cryptosporidium hominis and C. parvum reveals major knowledge gaps and a need for the application of "next generation" technologiesresearch review. Biotechnol Adv 28: 17-26.

Jothikumar N, da Silva AJ, Moura I, Qvarnstrom Y, Hill VR 2008. Detection and differentiation of Cryptosporidium hominis and Cryptosporidium parvum by dual TaqMan assays. J Med Microbiol 57: 1099-1105.

Leav BA, Mackay MR, Anyanwu A, O'Connor RM, Cevallos AM, Kindra G, Rollins NC, Bennish ML, Nelson RG, Ward HD 2002. Analysis of sequence diversity at the highly polymorphic Cpgp40/15 locus among Cryptosporidium isolates from human immunodeficiency virus-infected children in South Africa. Infect Immun 70: 3881-3890.

Li N, Xiao L, Cama VA, Ortega Y, Gilman RH, Guo M, Feng Y 2013. Genetic recombination and Cryptosporidium hominis virulent subtype IbA10G2. Emerg Infect Dis 19: 1573-1582.

Liu H, Shen Y, Yin J, Yuan Z, Jiang Y, Xu Y, Pan W, Hu Y, Cao J 2014. Prevalence and genetic characterization of Cryptosporidium, Enterocytozoon, Giardia, and Cyclospora in diarrheal outpatients in China. BMC Infect Dis 14: 1-6. 
Ma P, Soave R 1983. Three-step stool examination for cryptosporidiosis in 10 homosexual men with protracted watery diarrhea. $J$ Infect Dis 147: 824-828.

Meireles MV 2010. Cryptosporidium infection in Brazil: implications for veterinary medicine and public health. Rev Bras Parasitol Vet 19: 197-204.

Mohammad RZ, Ah JM, Mostafa R, Ahmed RM, Siavash V, Minoo M 2004. Prevalence of intestinal parasitic pathogens among HIVpositive individuals in Iran. Jpn J Infect Dis 57: 268-270.

Moura H, da Silva JL, Sodré FC, Brasil P, Wallmo K, Wahlquist S, Wallace S, Croppo GP, Visvesvara GS 1996. Gram-chromotrope: a new technique that enhances detection of microsporidial spores in clinical samples. $J$ Eukaryot Microbiol 43 (Suppl.): 94S-95S.

Moura H, Fernandes O, Viola JPB, Silva SP, Passos RH, Lima DB 1989. Enteric parasites and HIV infection: ocurrence in AIDS patients in Rio de Janeiro, Brazil. Mem Inst Oswaldo Cruz 84: 527-533.

Muthusamy D, Rao SS, Ramani S, Monica B, Banerjee I, Abraham OC, Mathai DC, Primrose B, Muliyil J, Wanke CA, Ward HD, Kang G 2006. Multilocus genotyping of Cryptosporidium sp. isolates from human immunodeficiency virus-infected individuals in South India. J Clin Microbiol 44: 632-634.

Navarro-i-Martinez L, del Águila C, Bornay-Llinares FJ 2011. Cryptosporidium: a genus in revision. The situation in Spain. Enferm Infecc Microbiol Clin 29: 135-143.

Nichols GL, Chalmers RM, Hadfield SJ 2014. Molecular epidemiology of human cryptospridiosis. In SM Cacciò, G Widmer (eds.), Cryptosporidium: parasite and disease, Springer, London, p. 81-148.

Peng MM, Meshnick SR, Cunliffe NA, Thindwa BD, Hart CA, Broadhead RL, Xiao L 2003. Molecular epidemiology of cryptosporidiosis in children in Malawi. J Eukaryot Microbiol 50 (Suppl.): 557-559.

Putignani L, Menichella D 2010. Global distribution, public health, and clinical impact of the protozoan pathogen cryptosporidium. Interdiscip Perspect Infect Dis 2010: 1-39.

Ritchie LS 1948. An ether sedimentation technique for routine stool examinations. Bull US Army Med Dep 8: 326.

Rolando RFR, da Silva S, Peralta RHS, da Silva AJ, Cunha FS, Bello AR, Peralta JM 2012. Detection and differentiation of Cryptosporidium by real-time polymerase chain reaction in stool samples from patients in Rio de Janeiro, Brazil. Mem Inst Oswaldo Cruz 107: 476-479.

Ryan U, Fayer R, Xiao L 2014. Cryptosporidium species in humans and animals: current understanding and research needs. Parasitology 141: 1667-1685.

Ryan U, Hijjawi N 2015. New developments in Cryptosporidium research. Int J Parasitol 45: 367-373.

Segura R, Prim N, Montemayor M, Valls ME, Munoz C 2015. Predominant virulent IbA10G2 subtype of Cryptosporidium hominis in human isolates in Barcelona: a five-year study. PLOS ONE 10: $\mathrm{e} 0121753$.

Sharma P, Sharma A, Sehgal R, Malla N, Khurana S 2013. Genetic diversity of Cryptosporidium isolates from patients in north India. Int J Infect Dis 17: e601-605.
Strong WB, Gut J, Nelson RG 2000. Cloning and sequence analysis of a highly polymorphic Cryptosporidium parvum gene encoding a 60-kilodalton glycoprotein and characterization of its 15 and 45-kilodalton zoite surface antigen products. Infect Immun 68: 4117-4134.

Sulaiman IM, Lal AA, Xiao L 2001. A population genetic study of the Cryptosporidium parvum human genotype parasites. J Eukaryot Microbiol 48 (Suppl. 1): 24S-27S.

Valenzuela O, González-Díaz M, Garibay-Escobar A, Burgara-Estrella A, Cano M, Durazo M, Bernal RM, Hernandez J, Xiao L 2014. Molecular characterization of Cryptosporidium spp in children from Mexico. PLoS ONE 9: e96128.

Velásquez JN, Carnevale S, Corti M, Di Risio C, Bramajo J, Pinto M, Labbé JH, Chertcoff A, Cabrera M, Rodríguez M, Guarnera EA 1997. Classic strategies for diagnosis of cryptosporidiosis in patients with AIDS and chronic diarrhea. Acta Gastroenterol Latinoam 27: 107-111.

Velásquez JN, Di Risio C, Marta E, Astudillo OG, Etchart C, Cucher MA, Chertcoff AV, Perissé E, Carnevale S 2010. Occurrence of bile-duct/duodenal abnormalities in nine AIDS patients coinfected with Cryptosporidium hominis and/or C. parvum. Ann Trop Med Parasitol 104: 257-263.

Weber R, Bryan RT, Owen RL, Wilcox CM, Gorelkin L, Visvesvara GS 1992. Improved light-microscopical detection of microsporidia spores in stool and duodenal aspirates. The Enteric Opportunistic Infections Working Group. N Engl J Med 326: 161-166.

Widerström M, Schönning C, Lilja M, Lebbad M, Ljung T, Allestam G, Ferm M, Björkholm B, Hansen A, Hiltula J, Långmark J, Löfdahl M, Omberg M, Reuterwall C, Samuelsson E, Widgren K, Wallensten A, Lindh J 2014. Large outbreak of Cryptosporidium hominis infection transmitted through the public water supply, Sweden. Emerg Infect Dis 20: 581-589.

Widmer G, Sullivan S 2012. Genomics and population biology of Cryptosporidium species. Parasite Immunol 34: 61-71.

Wu Z, Nagano I, Boonmars T, Nakada T, Takahashi Y 2003. Intraspecies polymorphism of Cryptosporidium parvum revealed by PCR-restriction fragment length polymorphism (RFLP) and RFLP-single-strand conformational polymorphism analyses. Appl Environ Microbiol 69: 4720-4726.

Xiao L 2010. Molecular epidemiology of cryptosporidiosis: an update. Exp Parasitol 124: 80-89.

Xiao L, Feng Y 2008. Zoonotic cryptosporidiosis. FEMS Immunol Med Microbiol 52: 309-323.

Xiao L, Ryan UM 2004. Cryptosporidiosis: an update in molecular epidemiology. Curr Opin Infect Dis 17: 483-490.

Yang R, Murphy C, Song Y, Ng-Hublin J, Estcourt A, Hijjawi N, Chalmers R, Hadfield S, Bath A, Gordon C, Ryan U 2013. Specific and quantitative detection and identification of Cryptosporidium hominis and C. parvum in clinical and environmental samples. Exp Parasitol 135: 142-147.

Zaidah AR, Chan YY, Asma HS, Abdullah S, Nurhaslindawati AR, Salleh M, Zeehaida M, Lalitha P, Mustafa M, Ravichandran M 2008. Detection of Cryptosporidium parvum in HIV-infected patients in Malaysia using a molecular approach. Southeast Asian J Trop Med Public Health 39: 511-516. 